\title{
Online Washing Process Monitoring with Wireless Textile Impedance Measurement
}

\author{
Andreas Hennig', Hartmut Prinz ${ }^{2}$, Mathias Roth ${ }^{3}$, Felix Essinghold ${ }^{1}$, Lukas Krupp ${ }^{1}$, \\ Anton Grabmaier ${ }^{1}$, Hans-Günter Hloch ${ }^{2}$, Jürgen Bohnen ${ }^{2}$ \\ ${ }^{1}$ Fraunhofer IMS, Germany, ${ }^{2}$ wfk - Cleaning Technology Institute e.V., Germany, \\ ${ }^{3}$ Textilforschungsinstitut Thüringen-Vogtland, Germany \\ andreas.hennig@ims.fraunhofer.de
}

\begin{abstract}
Summary:
In this paper a sensor system for the in-situ monitoring of dirt removal in the running washing process is presented. In this work, a sensor is presented for adjustment or optimization of washing processes in textile service companies. The developed impedimetric performance sensor consists of a dirt and reference sensor area as well as a measuring and RFID communication module. At the sensor areas the impedance is measured during the washing process, recorded and transferred to an external evaluation unit. The impedance values can be used to determine the dirt removal during the washing process.
\end{abstract}

Keywords: washing process monitoring, RFID sensor, impedimetric sensor

\section{Introduction}

In Germany, 1.8 million tons of textiles for professional use, are processed by service companies every year with a turnover of about 3.2 billion $€$. Due to constantly increasing customer requirements regarding the cleanliness and hygiene of textiles, up to $2.5 \%$ of the processed textiles (depending on the type of textile or laundry) are sorted out within the scope of quality control. On the other hand, excessive use of heat and detergents, bleaches or disinfectants leads to increased energy costs and premature damage to textiles. Hence there is a need to adjust and optimize the washing process at regular intervals or permanently in a feedback-loop.

\section{State-of-the-Art}

Today primary wash monitors are used. These consist of defined soiled test textiles, which are colorimetrically evaluated after the washing process. However, an evaluation of the washing process is only possible afterwards. Furthermore, no direct conclusions on the effect of the process parameters are possible, so that an iterative adjustment is necessary. The process is therefore laborious and complex for the user.

An RFID module including an impedimetric performance sensor shall enable the in situ online acquisition of relevant process parameters.

\section{Concept}

The concept provides an in-situ detection of the dirt removal via impedance measurements and online data transfer during a running washing process by means of RFID technology. Essential characteristics are:

- The sensor should pass through the process with the laundry items and behave like a textile in its mechanical properties

- The dirt removal is to be measured by recording the residual dirt on a sensor area

- Measurement data transmission should be feasible out of the washing machine drum.

The impedimetric performance sensor consists of a sensor area and a RFID transponder with a textile-based antenna. The in-situ detection of the dirt removal enables the identification of functional correlations between the dirt removal and the process parameters (e.g. drum drive, chemical dosing; temperature; time) during the running washing process.

\section{Sensor Principle}

The sensor area is based on a textile electrode structure, which is soiled with a practice relevant type of dirt. This textile electrode structure consists of electrically conductive yarns (textile electrodes) included in a textile matrix. The geometry of the textile electrodes determines the measuring accuracy and response threshold to be achieved. The dirt load dependent dielectricity of the textile electrode structure (residual dirt on the sensor area of the impedimetric performance sensor) is detected by impedance measurements. 


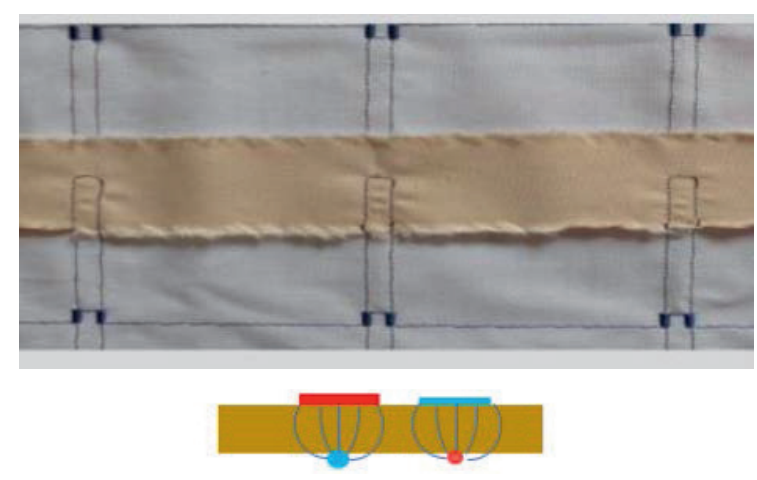

Fig. 1. Electrode structure and sensor area

Different electrode structures were evaluated. Figure 1 shows the chosen structure were higher proportion of the impedance signal comes from the textile and is therefore influenced by the contamination, compared to other arrangements. At the same time, the total value of the impedance is kept at a minimum level. Since the impedance must not be too low for the measuring circuit of the RFID module. The optimal sensor area consists of an electrode structure with electrodes that are guided at right angles to each other and attached to both sides of the soiled textile. The electrodes cross over each other at two points, so that the total impedance is still sufficiently high. Due to the improved electrode structure, commercially soiled textiles as well as specially produced soiled textiles could be used for the sensor area.

\section{Measuring Circuit and Data Transmission}

An RFID-module had to be developed, that performs the impedance measurement and enables wireless data transmission from the washing machine drum. Evaluation of textile electrodes have shown, that the impedance ranges from $10 \Omega$ to $500 \Omega$, when excited by a measurement frequency of $1 \mathrm{kHz}$.

The RFID-Module is composed of an integrated impedance converter circuit from analog devices (AD5933), which performs the impedance measurement, a microcontroller and a semi-passive RFID-Frontend ASIC and a textile dipole antenna working in the UHF-RFID-Band. The dipole antenna is realized using an embroidered conductive yarn. The electronic components were encapsulated with "Silikonharz Elastosil RT 646". The flexible textile antenna is connected via inductive coupling with a loop within the encapsulation. This allows the size of the rigid RFID module to be reduced.

To assess the feasibility, a reader antenna was mounted in the glass door of a front loading machine and the communication with a test transponder placed inside the washing machine was checked.

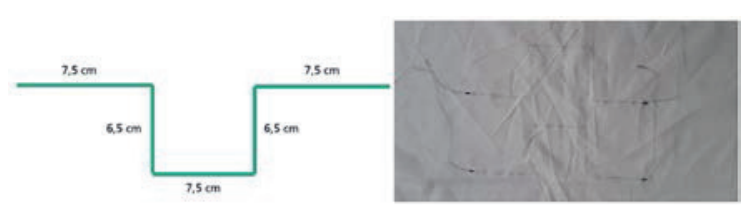

Fig. 2. Textile RFID antenna.

\section{Results}

To verify the measuring principle, the impedimetric sensor was placed in a washer extractor. The impedance measurement was performed at a frequency of $1 \mathrm{kHz}$.

Experiments with the impedimetric performance sensor in the washing machine during a washing program with a low mechanical agitation and a high liquor level (wool washing program) were done. A measurement frequency of $1 \mathrm{kHz}$ was used for the impedance measurement.

Figure 3 shows the impedance over time. A percentile filter was used for averaging in order to remove disturbances caused by the environment, especially the wash liquor, different soaking or covering of the sensor areas by other items of the laundry batch.

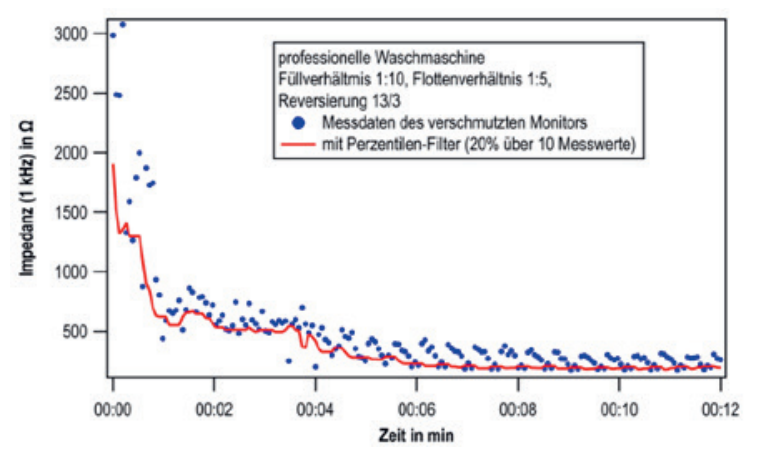

Fig. 3. Impedance curve during the washing process.

In summary, the impedimetric performance sensor allows the monitoring of the washing process and can be used to adjust temperature and concentration of the detergent or bleaching agent. In contrast to conventional wash monitors, there is no need for time-staggered removal and drying, which makes the readjustment of wash processes easier and faster.

It could be shown that inductively coupled dipole antennas can be realized with the help of conductive yarns. It could also be shown that RFID transponders can be read in metallic environments like a washing machine.

The IGF-project 19950 BG of the research as-sociation Forschungskuratorium Textil e.V., Reinhardtstr. 14-16, D-10177 Berlin, was supported via the AiF within the funding program ,Industrielle Gemeinschaftsforschung und -entwicklung (IGF)" by the Federal Ministry of Economic Affairs and Energy (BMWi) due to a decision of the German Parliament. 